\title{
Efficient Exciplex-Based Deep-Blue Organic Light-Emitting Diodes Employing a Bis(4-fluorophenyl)amine-Substituted Heptazine Acceptor
}

\author{
Jie Li ${ }^{1} \oplus$, Heqi Gong ${ }^{1}$, Jincheng Zhang ${ }^{1}$, Hui Liu ${ }^{1}$, Li Tao ${ }^{1}$, Yanqing Wang ${ }^{2}$ and Qiang Guo ${ }^{1, *}$ \\ 1 College of Optoelectronic Engineering, Chengdu University of Information Technology, \\ Chengdu 610225, China; lijie@cuit.edu.cn (J.L.); 3201105022@stu.cuit.edu.cn (H.G.); \\ 3201105004@stu.cuit.edu.cn (J.Z.); liuhui@cuit.edu.cn (H.L.); taoli@cuit.edu.cn (L.T.) \\ 2 College of Polymer Science and Engineering, Sichuan University, Chengdu 610065, China; \\ yanqingwang@scu.edu.cn \\ * Correspondence: qiangguo@cuit.edu.cn; Tel.: +86-28-8596-6385
}

check for updates

Citation: Li, J.; Gong, H.; Zhang, J.; Liu, H.; Tao, L.; Wang, Y.; Guo, Q. Efficient Exciplex-Based Deep-Blue Organic Light-Emitting Diodes Employing a Bis(4-fluorophenyl)amineSubstituted Heptazine Acceptor. Molecules 2021, 26, 5568. https:// doi.org/10.3390/molecules26185568

Academic Editors: Guohua Xie Chien-Jung Huang and Kuan-Wei Lee

Received: 24 July 2021

Accepted: 10 September 2021

Published: 13 September 2021

Publisher's Note: MDPI stays neutral with regard to jurisdictional claims in published maps and institutional affiliations.

Copyright: (C) 2021 by the authors Licensee MDPI, Basel, Switzerland. This article is an open access article distributed under the terms and conditions of the Creative Commons Attribution (CC BY) license (https:// creativecommons.org/licenses/by/ $4.0 /)$.

\begin{abstract}
The realization of a deep-blue-emitting exciplex system is a herculean task in the field of organic light-emitting diodes (OLEDs) on account of a large red-shifted and broadened exciplex emission spectrum in comparison to those of the corresponding single compounds. Herein, 2,5,8tris(di(4-fluorophenyl)amine)-1,3,4,6,7,9,9b-heptaazaphenalene (HAP-3FDPA) was designed as an electron acceptor by integrating three bis(4-fluorophenyl)amine groups into a heptazine core, while 1,3-di(9H-carbazol-9-yl)benzene ( $\mathrm{mCP}$ ) possessing two electron-donating carbazole moieties was chosen as the electron donor. Excitingly, the exciplex system of $8 w t \%$ HAP-3FDPA:mCP exhibited deep-blue emission and a high photoluminescence quantum yield of 53.2\%. More importantly, an OLED containing this exciplex system as an emitting layer showed deep-blue emission with Commission Internationale de $\mathrm{l}^{\prime}$ Eclairage coordinates of $(0.16,0.12)$, a peak luminance of $15,148 \mathrm{~cd} \mathrm{~m}^{-2}$, and a rather high maximum external quantum efficiency of $10.2 \%$ along with a low roll-off. This study not only reports an efficient exciplex-based deep-blue emitter but also presents a feasible pathway to construct highly efficient deep-blue OLEDs based on exciplex systems.
\end{abstract}

Keywords: exciplex; deep-blue; organic light-emitting diode; thermally activated delayed fluorescence; heptazine

\section{Introduction}

Organic light-emitting diodes (OLEDs) based on thermally activated delayed fluorescent (TADF) emitters have obtained considerable progress over the last decade [1-5]. As the third-generation organic light-emitting materials, TADF emitters possessing small singlet-triplet energy splitting $\left(\Delta E_{\mathrm{ST}}\right)$ between the lowest excited singlet state $\left(\mathrm{S}_{1}\right)$ and the lowest excited triplet state $\left(\mathrm{T}_{1}\right)$ can harvest both singlet and triplet excitons by efficient up-conversion from $T_{1}$ to $S_{1}$ through a reverse intersystem crossing (RISC) process [6-8]. To realize a small $\Delta E_{\mathrm{ST}}$, effective separation of electron densities of the highest occupied molecular orbital (HOMO) and the lowest unoccupied molecular orbital (LUMO) is indispensable because the $\Delta E_{\mathrm{ST}}$ is proportional to the exchange integrals between the wave functions of the HOMO and LUMO [9]. To date, several molecular design strategies have been proposed to achieve a small $\Delta E_{\mathrm{ST}}$, such as intramolecular $\pi \rightarrow \pi^{*}$ or $\mathrm{n} \rightarrow \pi^{*}$ charge transfer in a single molecule [10-15] and exciplex-based intermolecular charge transfer between an electron-donating molecule and an electron-accepting molecule [16-20].

On the basis of the natural TADF characteristics, the development of exciplex-based TADF emitters for OLEDs has attracted much attention in recent years. Formed between different molecules, the HOMO and LUMO of an exciplex system are naturally located on the electron-donating and electron-accepting molecules, respectively, resulting in almost 
complete separation and an extremely small $\Delta E_{\mathrm{ST}}$. Since the innovative work of TADF characteristics of exciplexes in 2012 by Adachi et al. [16], a large number of exciplex-based emitters have been developed, whereas a majority of them exhibit green, yellow, or red emission because exciplex formation is usually accompanied by a large red-shift and a broadened structure of the emission spectrum [20]. Accordingly, exciplex-based deepblue emitters are quite difficult to acquire and in urgent need of exciplex-based full-color displays or white-light OLEDs.

In this study, we developed an efficient deep-blue-emitting exciplex system employing 2,5,8-tris(di(4-fluorophenyl)amine)-1,3,4,6,7,9,9b-heptaazaphenalene (HAP-3FDPA), and 1,3-di(9H-carbazol-9-yl)benzene (mCP). HAP-3FDPA, which is composed of a heptazine core and three bis(4-fluorophenyl)amine groups, was designed as an electron acceptor, while $\mathrm{mCP}$ possessing two electron-donating carbazole moieties is a widely used host material [21] and was chosen as an electron donor to form exciplex with HAP-3FDPA. Herein, the heptazine core with a planar and rigid heterocyclic system of six $\mathrm{C}=\mathrm{N}$ bonds surrounding a central $\mathrm{sp}^{2}$-hybridised $\mathrm{N}$-atom was chosen as an ideal strong electronaccepting constituent [22-26]. Meanwhile, the three bis(4-fluorophenyl)amine groups were introduced to maintain the electron-withdrawing ability and to increase the solubility of the heptazine derivative. Excitingly, benefitting from the relatively rigid and planar molecular skeletons and strong charge transfer characteristics between HAP-3FDPA and mCP, the OLED incorporating an $8 w t \%$ HAP-3FDPA:mCP exciplex system exhibited deep-blue emission with Commission Internationale de l'Eclairage (CIE) coordinates of $(0.16,0.12)$ and a rather high maximum external quantum efficiency (EQE) of 10.2\% along with a fairly low roll-off at high luminance.

\section{Results and Discussion}

The chemical structure and synthetic route of HAP-3FDPA are depicted in Scheme 1. The target compound of HAP-3FDPA was synthesized by cyameluric chloride and bis(4fluorophenyl)amine. Thereinto, cyameluric chloride is the key intermediate and was prepared according to the literature [22]. Noteworthily, there is a low yield of $29 \%$ for HAP-3FDPA owing to the electron-withdrawing ability of fluorine atoms in bis(4fluorophenyl)amine. The target compound was characterized and confirmed via ${ }^{1} \mathrm{H}$ NMR, ${ }^{13} \mathrm{C}$ NMR spectroscopy, and a high-resolution mass spectrometer (HRMS).<smiles>Fc1ccc(N(C2=Nc3nc(nc(N(c4ccc(F)cc4)c4ccc(F)cc4)n3)N3C(=N2)N=C3N(c2ccc(F)cc2)c2ccc(N(c3ccc(F)cc3)c3ccc(F)cc3)cc2)c2ccc(F)cc2)cc1</smiles>

Scheme 1. Synthetic route of HAP-3FDPA.

To obtain insight into the electronic structure of HAP-3FDPA, quantum chemical calculations were carried out. The characteristics of molecular configuration, frontier orbitals, and the resulting HOMO and LUMO energy levels were obtained based on density functional theory (DFT) calculation, while the electron transition and excited energy levels of $S_{1}$ and $T_{1}$ were performed by time-dependent density functional theory (TD-DFT) analysis. These results are important to the photophysical properties of HAP3FDPA and can also provide a theoretical basis for the design of OLED structures. As depicted in Figures 1 and S1 (in Supplementary Materials), the optimized ground state 
structure revealed that HAP-3FDPA has relatively large dihedral angles of $56-59^{\circ}$ between the twisted bis(4-fluorophenyl)amine units and the planar heptazine core, together with that, the HOMO and LUMO are mainly distributed over the bis(4-fluorophenyl)amine units and the heptazine core, respectively. Furthermore, the obvious charge transfer character and the small overlap between the HOMO and LUMO leads to a small $\Delta E_{\mathrm{ST}}$ of $0.29 \mathrm{eV}$. The calculated HOMO and LUMO levels for HAP-3FDPA are -5.90 and $-1.64 \mathrm{eV}$, respectively. Herein, the deep HOMO and shallow LUMO energy levels should be ascribed to the weak electron-donating ability of bis(4-fluorophenyl)amine units and strong electron-accepting ability of the heptazine core, respectively. Meanwhile, it should be noted that the natural transition orbitals (NTOs) for $\mathrm{S}_{1}$ of HAP-3FDPA (HOMO-3 to LUMO) are deriving from localized $n \rightarrow \pi^{*}$ transitions involving lone-pair electrons of $\mathrm{N}$ heteroatoms and adjacent $\pi$ antibonding molecular orbitals (Figure S2), while the NTOs for $\mathrm{T}_{1}$ (HOMO to LUMO or $\mathrm{HOMO}-1$ to LUMO) have the more delocalized $\pi \rightarrow \pi^{*}$ transition characters (Figure S3).

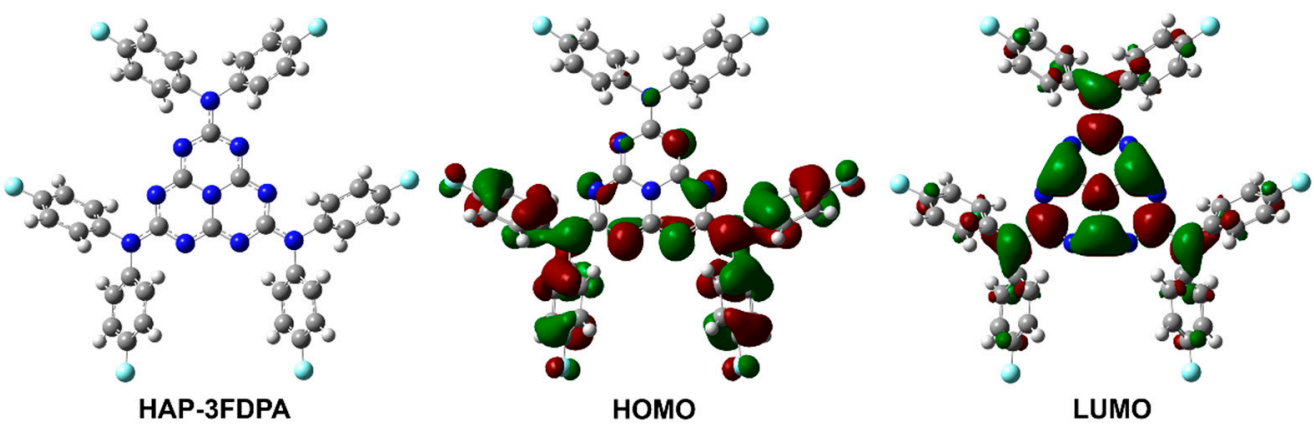

Figure 1. The optimized molecular geometry of the ground state and frontier molecular orbital distributions of HAP-3FDPA by theoretical calculations.

The HOMO energy level was measured by atmospheric ultraviolet photoelectron spectroscopy. As depicted in Figure 2a and Table 1, the HOMO energy level of HAP-3FDPA was determined to be $-6.1 \mathrm{eV}$. As calculated from the $\mathrm{HOMO}$ level and the optical energy gap $\left(E_{\mathrm{g}}=3.2 \mathrm{eV}\right.$, Figure $\left.2 \mathrm{~b}\right)$, the LUMO energy level was calculated to be $-2.9 \mathrm{eV}$. The ultraviolet-visible (UV) absorption and photoluminescence (PL) spectra of HAP-3FDPA in a neat film are shown in Figure $2 \mathrm{~b}$. The intense absorption band with a maximal absorption wavelength $\left(\lambda_{\text {abs }}\right)$ of $268 \mathrm{~nm}$ can be assigned to $\pi \rightarrow \pi^{*}$ electronic transition in view of the $\pi$-conjugated molecular system. Meanwhile, HAP-3FDPA in the neat film displayed skyblue emission with an emission peak wavelength $\left(\lambda_{\mathrm{em}}\right)$ of $464 \mathrm{~nm}$. The UV and PL spectra of HAP-3FDPA in dilute toluene at a concentration of $1 \times 10^{-4} \mathrm{~mol} \mathrm{~L}^{-1}$ are shown in Figure 2c. Similar to that of HAP-3FDPA in a neat film, the strong absorption band centered at $319 \mathrm{~nm}$ should be attributed to $\pi \rightarrow \pi^{*}$ electronic transition. Interestingly, HAP-3FDPA in toluene displayed green emission with $\lambda_{\mathrm{em}}=533 \mathrm{~nm}$, indicating that there was a large molecular geometry variation of HAP-3FDPA in a toluene condition in comparison to that in a neat film. Moreover, transient PL decay of HAP-3FDPA both in air-saturated and in oxygen-free toluene was measured (Figure $2 \mathrm{~d}$ ). Apparently, only one prompt component decay could be observed with the lifetime of prompt emission $\left(\tau_{\mathrm{p}}\right)$ of $1.9 \mathrm{~ns}$. Meanwhile, a quite low PL quantum yield (PLQY) of 5.1\% was recorded in both air-saturated and oxygen-free toluene. Consequently, the radiative rate constant of fluorescence $\left(k_{\mathrm{F}}\right)$ of HAP3FDPA was calculated to be $2.7 \times 10^{7} \mathrm{~s}^{-1}$ according to the equation of $k_{\mathrm{F}}=\mathrm{PLQY} / \tau_{\mathrm{p}}$. Thus, the oxygen-independent transient PL decay and PLQY indicate the absence of delayed fluorescence, which probably originated from the whole electron-withdrawing molecular structure of HAP-3FDPA. 

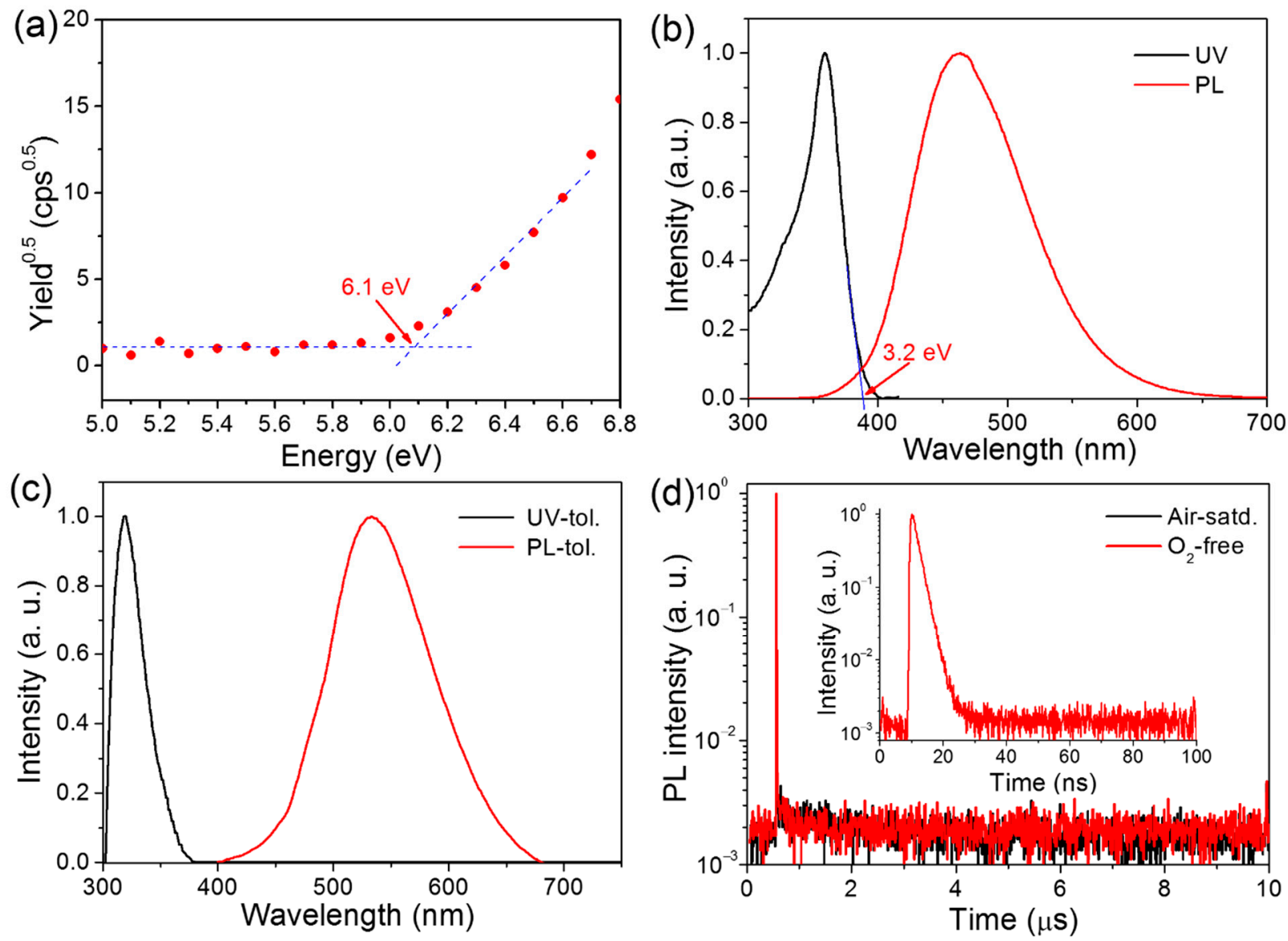

Figure 2. (a) The HOMO energy level of HAP-3FDPA determined by atmospheric ultraviolet photoelectron spectroscopy. (b) The UV and PL spectra of HAP-3FDPA in a neat film. (c) The UV and PL spectra of HAP-3FDPA in diluted toluene (tol.). (d) Transient PL decay of HAP-3FDPA in air-saturated (Air-satd.) and oxygen-free $\left(\mathrm{O}_{2}\right.$-free) toluene. Inset: transient PL decay in a time range of $100 \mathrm{~ns}$.

Table 1. The photophysical properties of HAP-3FDPA and an $8 w t \%$ HAP-3FDPA:mCP-doped film.

\begin{tabular}{cccccccc}
\hline Compound & HOMO/LUMO (eV) & $\lambda_{\text {abs }}(\mathbf{n m})$ & $\lambda_{\text {em }}(\mathbf{n m})$ & $\tau_{\mathbf{p}}(\mathbf{n s})$ & $\tau_{\mathbf{d}}(\mathbf{n s})$ & PLQY & FWHM $(\mathbf{n m})$ \\
\hline HAP-3FDPA & $-6.1 /-2.9$ & $359^{\mathrm{a}} / 319^{\mathrm{b}}$ & $464^{\mathrm{a}} / 533^{\mathrm{b}}$ & $1.9^{\mathrm{b}}$ & - & $5.1 \% \mathrm{~b}$ & $101^{\mathrm{a}} / 102^{\mathrm{b}}$ \\
Doped film & - & 342 & 433 & 3.0 & $8.0,86.1$ & $53.2 \%$ & 87 \\
\hline
\end{tabular}

${ }^{\mathrm{a}}$ Measured in a neat film; ${ }^{\mathrm{b}}$ measured in oxygen-free toluene.

To verify that HAP-3FDPA can be used as an electron acceptor in the exciplex system, 1,3-di(9H-carbazol-9-yl)benzene ( $\mathrm{mCP}$ ) possessing two electron-donating carbazole moieties and a high $\mathrm{T}_{1}$ level is a popular host material and was chosen as the electron donor to form exciplex with HAP-3FDPA [21]. Subsequently, an $8 w t \%$ HAP-3FDPA:mCP-doped film was fabricated and characterized, and the photophysical properties are exhibited in Figure 3 and Table 1 . Herein, the concentration of $8 w t \%$ was chosen based on the optimization of luminescence efficiencies at various concentrations (Table S1). Most strikingly, the $8 w t \%$ HAP-3FDPA:mCP-doped film showed deep-blue emission with $\lambda_{\mathrm{em}}=433 \mathrm{~nm}$ (Figure 3a), which is significantly blue-shifted compared to those in a neat film $(464 \mathrm{~nm})$ and toluene $(533 \mathrm{~nm})$. Meanwhile, the structureless and smooth emission spectrum presented a narrow full width at half maximum (FWHM) of $87 \mathrm{~nm}$ in comparison with those of HAP-3FDPA in a neat film $(101 \mathrm{~nm})$ and in diluted toluene $(102 \mathrm{~nm})$, which is beneficial to the improvement of color purity for exciplex systems. As we know, there is usually a red-shifted and broadened emission spectrum for exciplex systems compared to those of corresponding single compounds [27-32]. Therefore, a large number of green- and red-emitting exciplex systems have been developed, whereas deep-blue-emitting exciplex 
systems are quite difficult to realize [20]. Hence, the PL spectrum of the $8 w t \%$ HAP3FDPA:mCP-doped film is remarkably different from the emission tendency of traditional exciplex systems.
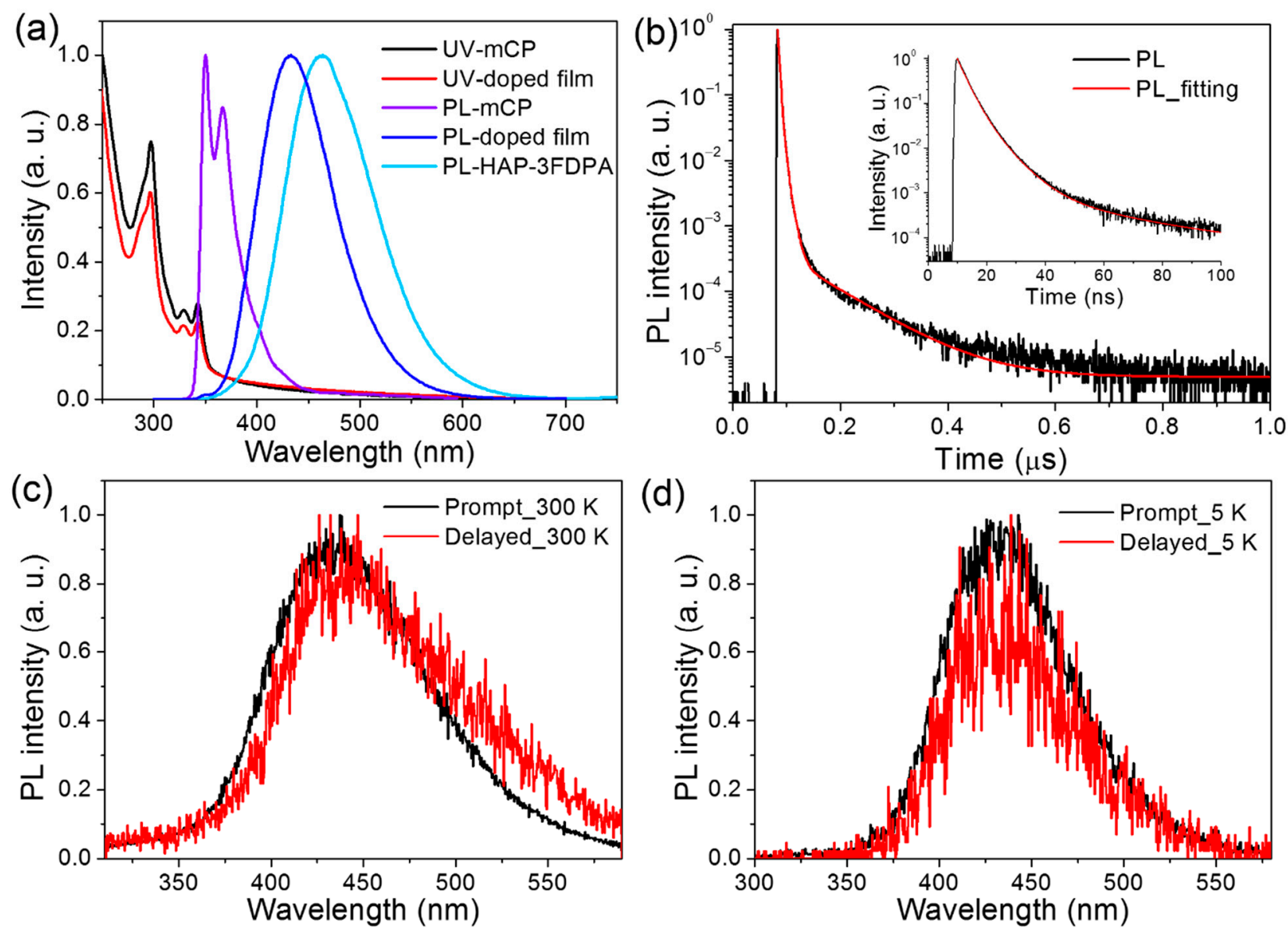

Figure 3. (a) The UV and PL spectra of $\mathrm{mCP}$, HAP-3FDPA in neat film, and $8 w t \%$ HAP-3FDPA:mCP-doped film at $300 \mathrm{~K}$. (b) Transient PL decay of the $8 w t \%$ HAP-3FDPA:mCP-doped film at $300 \mathrm{~K}$. Inset: transient PL decay with a time range of 100 ns. (c) Prompt and delayed PL spectra of the $8 w t \%$ HAP-3FDPA:mCP-doped film at 300 K. (d) Prompt and delayed PL spectra of the $8 w t \%$ HAP-3FDPA:mCP-doped film at $5 \mathrm{~K}$.

To confirm the exciplex emission of the $8 w t \%$ HAP-3FDPA:mCP-doped film, transient PL decay of the doped film at $300 \mathrm{~K}$ was measured and is shown in Figures $3 \mathrm{~b}$ and S4. Obviously, the decay process can be divided into prompt and delayed components. To better elucidate the exciplex emission, prompt and delayed emission spectra of the $8 w t \%$ HAP-3FDPA:mCP-doped film at both 300 and $5 \mathrm{~K}$ were characterized (Figure $3 c, d$ ). The well-overlapped prompt and delayed emission spectra at $300 \mathrm{~K}$ manifest that all photons are generated from the same excited state. Meanwhile, the considerably good overlap of fluorescence and phosphorescence spectra at $5 \mathrm{~K}$ confirms that the doped film possesses an extremely small $\Delta E_{\mathrm{ST}}$. Thus, the $8 w t \%$ HAP-3FDPA:mCP-doped film can be considered as an exciplex system. Additionally, the strong prompt component with $\tau_{\mathrm{p}}=3.0 \mathrm{~ns}$ should be assigned to exciplex-based fluorescence, while the weak delayed component with two comparably short lifetimes $\left(\tau_{\mathrm{d}}\right)$ of 8.0 and $86.1 \mathrm{~ns}$ can be attributed to the exciplexbased delayed fluorescence. It is noteworthy that the $8 w t \%$ HAP-3FDPA:mCP exciplex system displayed a relatively high PLQY of $53.2 \%$, which is much higher than that of HAP-3FDPA in dilute toluene $(5.1 \%)$, implying efficient radiative transition of singlet excitons from $S_{1}$ to the ground state. Furthermore, to elucidate the mechanism of exciplex emission, as shown in Figure S5, the HOMO and LUMO levels of $\mathrm{mCP}$ were measured to be -6.1 and $-2.6 \mathrm{eV}$ by atmospheric ultraviolet photoelectron spectroscopy and $\mathrm{UV}$ 
spectra, respectively. Obviously, the aforementioned deep-blue emission of the $8 w t \%$ HAP-3FDPA:mCP exciplex system should be attributed to the shallow LUMO $(-2.9 \mathrm{eV})$ of HAP-3FDPA and deep HOMO $(-6.1 \mathrm{eV})$ of $\mathrm{mCP}$, leading to a large energy gap of $3.2 \mathrm{eV}$ for the exciplex formation [16]. Meanwhile, the narrow FWHM may stem from the fairly rigid and planar geometries of HAP-3FDPA and $\mathrm{mCP}$, which tend to result in tight molecular packing and strong intermolecular interactions [18,33]. Additionally, blend films of $8 w t \%$ HAP-3FDPA:DPEPO (bis(2-(diphenylphosphino)phenyl) ether oxide) and $8 w t \%$ HAP-3FDPA:TCTA $\left(4,4^{\prime}, 4^{\prime \prime}\right.$-tris( $N$-carbazolyl)triphenylamine) were fabricated and compared with that of $8 w t \%$ HAP-3FDPA:mCP-doped film. As shown in Figures S6 and S7, no delayed component was observed in the transient PL decay of $8 w t \%$ HAP-3FDPA:DPEPO blend film, indicating the absence of exciplex emission due to the electron-accepting character of the DPEPO molecule. Meanwhile, the PL spectrum of the $8 w t \%$ HAP-3FDPA:TCTA blend film showed an apparent red-shift as compared to that of the $8 w t \%$ HAP-3FDPA:mCP-doped film, which should be ascribed to the shallower HOMO of TCTA than that of $\mathrm{MCP}$ (Scheme 2).

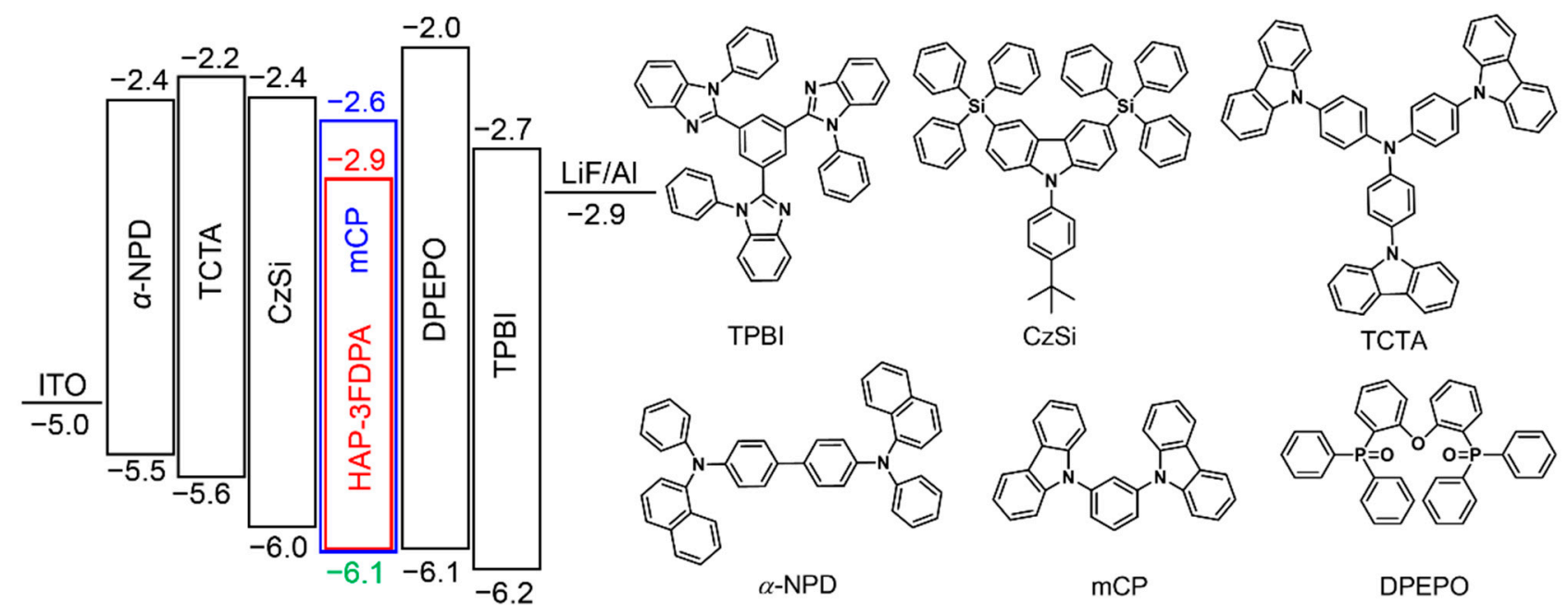

Scheme 2. The energy diagram of the HOMO and LUMO levels of materials utilized in the OLED device and corresponding chemical molecular structures.

To evaluate EL performance of the $8 w t \%$ HAP-3FDPA:mCP exciplex system, an OLED device incorporating an emitting layer of the exciplex system was fabricated with a structure of ITO (indium tin oxide) / $\alpha$-NPD ( $N, N^{\prime}$-diphenyl- $N, N^{\prime}$-bis(1-naphthyl)-1,10-biphenyl-4, $4^{\prime}$ diamine) $(30 \mathrm{~nm}) / \mathrm{TCTA}(20 \mathrm{~nm}) / \mathrm{CzSi}$ (9-(4-tert-butylphenyl)-3,6-bis(triphenylsilyl)-9Hcarbazole) $(10 \mathrm{~nm}) / 8 w t \%$ HAP-3FDPA:mCP $(20 \mathrm{~nm}) /$ DPEPO $(10 \mathrm{~nm}) /$ TPBI $(1,3,5$-tris $(N$ phenylbenzimidazol-2-yl)benzene) $(30 \mathrm{~nm}) / \mathrm{LiF}(1 \mathrm{~nm}) / \mathrm{Al}(100 \mathrm{~nm})$. The energy diagram and chemical molecular structures of organic compounds employed in the OLED device are depicted in Scheme 2. The EL spectra of this device measured at 1, 10,100 $\mathrm{mA} \mathrm{cm}^{-2}$ are welloverlapped with a maximum EL peak $\left(\lambda_{\mathrm{EL}}\right)$ of $437 \mathrm{~nm}$ and are similar to the PL spectrum of the emitting layer (Figure 4a). Meanwhile, the photo energy of the exciplex was calculated to be $3.2 \mathrm{eV}$ from the onset of the EL spectrum $(373 \mathrm{~nm})$, which is in good agreement with the energy difference between the LUMO of HAP-3FDPA and the HOMO of mCP (Figure S5). More importantly, the OLED showed deep-blue emission with CIE coordinates of $(0.16,0.12)$, a turn-on voltage $\left(V_{\text {on }}\right)$ of $4.0 \mathrm{~V}$, a peak luminance $\left(L_{\max }\right)$ of $15,148 \mathrm{~cd} \mathrm{~m}^{-2}$, and a rather high maximum external quantum efficiency (EQE) of $10.2 \%$ without any light out-coupling enhancement (Figure $4 \mathrm{~b}-\mathrm{d}$ and Table 2). Moreover, it is noteworthy that there is a rather low-efficiency roll-off at high luminance for the exciplex-based OLED, with $10.0 \%, 9.0 \%, 7.7 \%$, and $6.7 \%$ at $100,1000,5000$, and $10,000 \mathrm{~cd} \mathrm{~m}^{-2}$, respectively. The excellent EL performance of the OLED employing an $8 w t \%$ HAP-3FDPA:mCP exciplex 
system should be predominantly ascribed to efficient up-conversion of triplet excitons from $\mathrm{T}_{1}$ to $\mathrm{S}_{1}$ through the TADF process under electrical excitation.

(a)

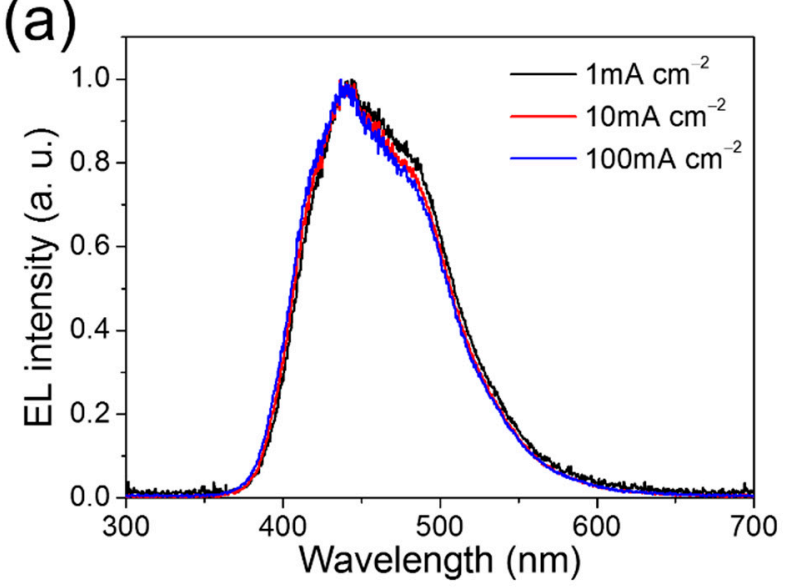

(c)

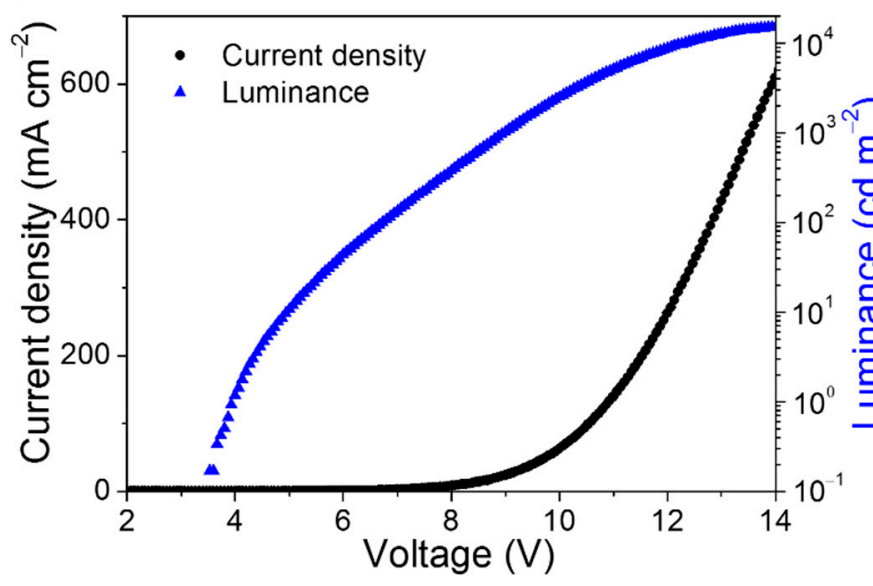

(b)

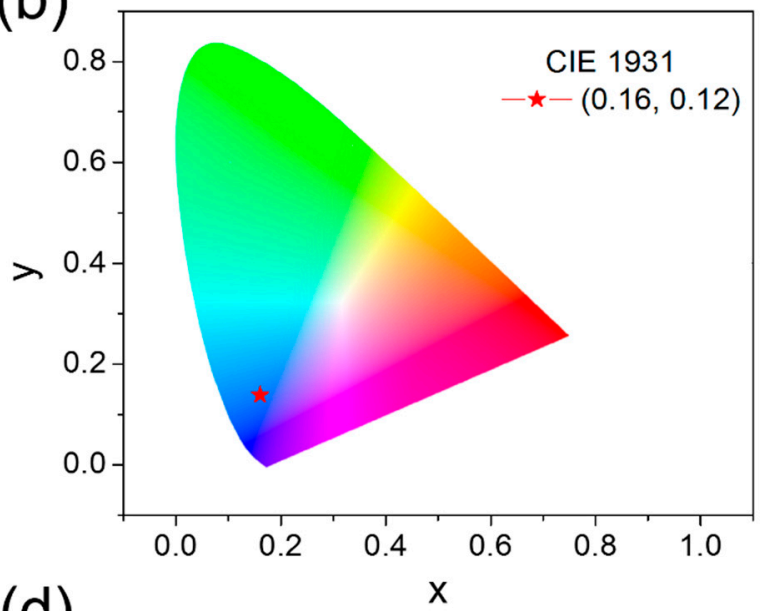

(d)

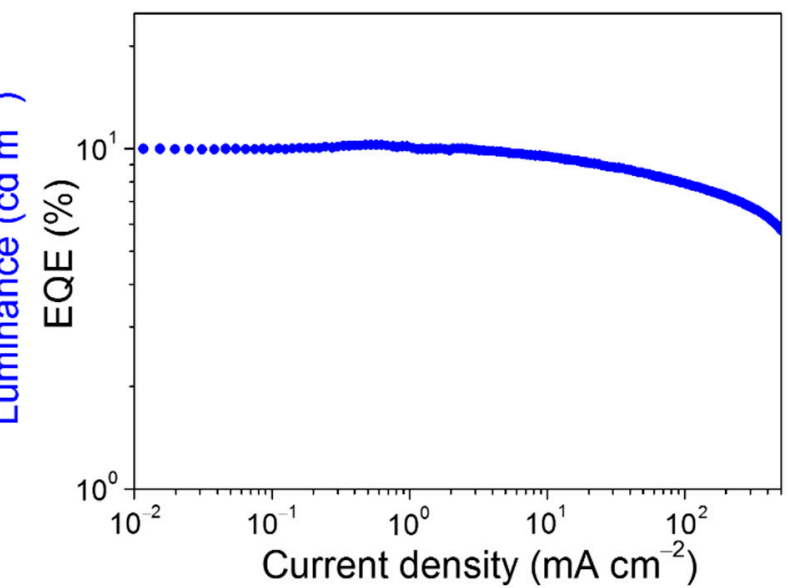

Figure 4. EL characteristics of the $8 w t \%$ HAP-3FDPA:mCP exciplex-based OLED. (a) The EL spectra at various current densities. (b) The CIE coordinates of $(0.16,0.12)$ for EL spectra. (c) The current efficiency-voltage-luminance $(J-V-L)$ characteristics. (d) EQE as a function of current density.

Table 2. The OLED performance based on an $8 w t \%$ HAP-3FDPA:mCP exciplex system.

\begin{tabular}{cccccc}
\hline Emitter & $V_{\text {on }}(\mathbf{V})^{\mathbf{a}}$ & $\lambda_{\text {EL }}(\mathbf{n m})$ & $L_{\max }\left(\mathbf{c d ~ \mathbf { m } ^ { - 2 } )}\right.$ & EQE $(\mathbf{\%})^{\mathbf{b}}$ & CIE $(\mathbf{x}, \mathbf{y})$ \\
\hline $8 w \%^{2}$ HAP-3FDPA:mCP & 4.0 & 437 & 15,148 & $10.2 / 10.0 / 9.0 / 7.7 / 6.7$ & $0.16,0.12$ \\
\hline
\end{tabular}

a Turn-on voltage at $1 \mathrm{~cd} \mathrm{~m}^{-2}, \mathrm{~b}$ the maximum EQE, EQEs at 100, 1000, 5000, and 10,000 $\mathrm{cd} \mathrm{m}^{-2}$, respectively.

\section{Materials and Methods}

3.1. Synthesis of 2,5,8-Tris(di(4-fluorophenyl)amine)-1,3,4,6,7,9,9b-heptaazaphenalene (HAP-3FDPA)

A flame-dried Schlenk tube with a magnetic stir bar was charged with mixture of cyameluric chloride (1.09 mmol, $300 \mathrm{mg})$, bis(4-fluorophenyl)amine (17.7 mmol, $3.6 \mathrm{~g}$ ) and dry xylene $(20 \mathrm{~mL})$ under an $\mathrm{N}_{2}$ atmosphere. The resulting mixture was heated at $180{ }^{\circ} \mathrm{C}$ for $24 \mathrm{~h}$. After cooling to room temperature, the solvent was removed by vacuum distillation. The residue was purified by column chromatography on silica gel and recrystallized from ethyl acetate/petroleum ether mixtures to provide the desired product as white solid (250 mg, 29\%). ${ }^{1} \mathrm{H}$ NMR $\left(400 \mathrm{MHz}, \mathrm{DMSO}-d_{6}\right): \delta(\mathrm{ppm})=7.28-7.34(\mathrm{~m}, 1 \mathrm{H})$, $7.17(\mathrm{t}, J=8.8 \mathrm{~Hz}, 1 \mathrm{H}) .{ }^{13} \mathrm{C}$ NMR $\left(100 \mathrm{MHz}, \mathrm{DMSO}-d_{6}\right): \delta(\mathrm{ppm})=164.38,161.67,159.23$, 
155.81, 139.22, 130.02, 129.94, 116.22, 115.99. HRMS (ESI ${ }^{+}$): calcd. for $\mathrm{C}_{42} \mathrm{H}_{25} \mathrm{~F}_{6} \mathrm{~N}_{10}[\mathrm{M}+\mathrm{H}]^{+}$ 783.2090, found 783.2092.

\subsection{OLED Fabrication and Measurement}

The OLED was fabricated by vacuum thermal evaporation under pressure lower than $5 \times 10^{-4} \mathrm{~Pa}$. A $150 \mathrm{~nm}$-thick indium-tin-oxide (ITO) precoated glass substrate was used as the anode. Prior to the deposition of the organic layers and cathode, the substrate was firstly cleaned with ultra-purified water, acetone, and isopropyl alcohol (IPA) in sequence, then treated with UV-ozone for $15 \mathrm{~min}$ and finally transferred to a vacuum thermal deposition system. The intersection of ITO and the metal electrodes gave an active device area of $4 \mathrm{~mm}^{2}$. The OLED device was characterized under atmospheric conditions without any encapsulation or light out-coupling enhancement. The EL spetrum, EQE, and current density-voltage-luminance $(J-V-L)$ characteristics of the OLED were recorded with a semiconductor parameter analyzer (E5270, Agilent, Santa Clara, CA, USA) and an optical power meter (1930C, Newport, Irvine, CA, USA). EL spectra were recorded using a multi-channel spectrometer (SD2000, Ocean Optics, Dunedin, FL, USA).

\section{Conclusions}

In summary, we designed an efficient exciplex-based deep-blue emitter incorporating 2,5,8-tris(di(4-fluorophenyl)amine)-1,3,4,6,7,9,9b-heptaazaphenalene (HAP-3FDPA) as the electron acceptor and 1,3-di $(9 H$-carbazol-9-yl)benzene $(\mathrm{mCP})$ as the electron donor. The $8 w t \%$ HAP-3FDPA:mCP exciplex system exhibited deep-blue emission with $\lambda_{\mathrm{em}}=433 \mathrm{~nm}$ and a fairly small $\Delta E_{\mathrm{ST}}$, giving rise to efficient exciton up-conversion and a high PLQY of $53.2 \%$. More importantly, an OLED containing this exciplex system as an emitting layer showed deep-blue emission with CIE coordinates of $(0.16,0.12)$, a peak luminance $\left(L_{\max }\right)$ of $15148 \mathrm{~cd} \mathrm{~m}^{-2}$, and a rather high maximum external quantum efficiency (EQE) of 10.2\% along with a fairly low roll-off at high luminance. These findings are of fundamental interest for the development of deep-blue OLEDs based on exciplex systems. Through the elaborate molecular design of electron donors and acceptors, we believe that highly efficient exciplex-based deep-blue OLEDs can be expected.

Supplementary Materials: Instrumentation; Quantum chemical calculations; Figure S1: Frontier molecular orbital distributions and energy levels of the lowest excited singlet and triplet states of HAP-3FDPA by theoretical calculations; Figure S2: The natural transition orbitals $(197 \rightarrow 201)$ for the lowest excited singlet state $\left(\mathrm{S}_{1}\right)$ of HAP-3FDPA by theoretical calculations; Figure S3: The natural transition orbitals $(199 \rightarrow 201)$ and $(200 \rightarrow 201)$ for the lowest excited triplet state $\left(\mathrm{T}_{1}\right)$ of HAP-3FDPA by theoretical calculations; Figure S4: The transient PL decay image of $8 w t \%$ HAP3FDPA:mCP-doped film in the time range of $2 \mu \mathrm{s}$; Figure S5: (a) The HOMO energy level of mCP determined by atmospheric ultraviolet photoelectron spectroscopy. (b) The UV spectra of mCP in a neat film. The optical energy gap of $\mathrm{mCP}$ was calculated to be $3.5 \mathrm{eV}$. Therefore, the LUMO energy level of $\mathrm{mCP}$ could be calculated to be $-2.6 \mathrm{eV}$; Figure S6: The transient PL decay of $8 w t \%$ HAP-3FDPA:DPEPO-doped film in the time range of $5 \mu$ s; Figure S7: The PL spectrum of $8 w t \%$ HAP-3FDPA:TCTA-doped film as compared to that of $8 w t \%$ HAP-3FDPA:mCP; Figure S8: The energy diagram of the $8 w t \%$ HAP-3FDPA:mCP exciplex system; Figure S9: ${ }^{1} \mathrm{H}$ NMR spectrum of HAP-3FDPA in DMSO- $d_{6}$; Figure S10: ${ }^{13} \mathrm{C}$ NMR spectrum of HAP-3FDPA in DMSO- $d_{6}$; Table S1: The PLQYs of HAP-3FDPA:mCP doped films at various concentrations.

Author Contributions: Conceptualization, J.L. and Q.G.; methodology, H.G. and J.Z.; software, H.G. and J.Z.; validation, J.L. and Q.G.; formal analysis, J.L. and Q.G.; investigation, J.L. and Q.G.; resources, H.L., L.T. and Y.W.; data curation, H.L., L.T. and Y.W.; writing-original draft preparation, J.L.; writing—review and editing, Q.G.; visualization, J.L. and Q.G.; supervision, H.L., L.T. and Y.W.; project administration, J.L. and Q.G.; funding acquisition, J.L. and Q.G. All authors have read and agreed to the published version of the manuscript.

Funding: This research was funded by the National Natural Science Foundation of China (grant numbers: 61505015, 21801028, 11704050), the Department of Science and Technology of Sichuan Province (grant numbers: 2019YJ0358, 2017FZ0085, 2020YFH0104), Fundamental Research Funds for 
the Central Universities (grant number: YJ201952), and the Department of Human Resources and Social Security of Sichuan Province (grant number: 2019Z226).

Institutional Review Board Statement: Not applicable.

Informed Consent Statement: Not applicable.

Data Availability Statement: The data presented in this study are available on request from the corresponding author.

Conflicts of Interest: The authors declare no conflict of interest.

\section{References}

1. Uoyama, H.; Goushi, K.; Shizu, K.; Nomura, H.; Adachi, C. Highly efficient organic light-emitting diodes from delayed fluorescence. Nature 2012, 492, 234-238. [CrossRef] [PubMed]

2. Konidena, R.K.; Lee, J.Y. Molecular design tactics for highly efficient thermally activated delayed fluorescence emitters for organic light emitting diodes. Chem. Rec. 2019, 19, 1499-1517. [CrossRef] [PubMed]

3. Cai, M.; Zhang, D.; Duan, L. High performance thermally activated delayed fluorescence sensitized organic light-emitting diodes. Chem. Rec. 2019, 19, 1611-1623. [CrossRef]

4. Kim, J.U.; Park, I.S.; Chan, C.Y.; Tanaka, M.; Tsuchiya, Y.; Nakanotani, H.; Adachi, C. Nanosecond-time-scale delayed fluorescence molecule for deep-blue oleds with small efficiency rolloff. Nat. Commun. 2020, 11, 1765. [CrossRef]

5. Peng, C.C.; Yang, S.Y.; Li, H.C.; Xie, G.H.; Cui, L.S.; Zou, S.N.; Poriel, C.; Jiang, Z.Q.; Liao, L.S. Highly efficient thermally activated delayed fluorescence via an unconjugated donor-acceptor system realizing eqe of over 30\%. Adv. Mater. 2020, $32,2003885$. [CrossRef] [PubMed]

6. Kim, M.; Jeon, S.K.; Hwang, S.H.; Lee, S.S.; Yu, E.; Lee, J.Y. Highly efficient and color tunable thermally activated delayed fluorescent emitters using a "twin emitter" molecular design. Chem. Commun. 2016, 52, 339-342. [CrossRef] [PubMed]

7. Lundberg, P.; Lindh, E.M.; Tang, S.; Edman, L. Toward efficient and metal-free emissive devices: A solution processed host guest light-emitting electrochemical cell featuring thermally activated delayed fluorescence. ACS Appl. Mater. Inter. 2017, 9, 28810-28816. [CrossRef]

8. Yin, X.; He, Y.; Wang, X.; Wu, Z.X.; Pang, E.B.; Xu, J.; Wang, J.A. Recent advances in thermally activated delayed fluorescent polymer-molecular designing strategies. Front. Chem. 2020, 8, 725. [CrossRef] [PubMed]

9. Endo, A.; Sato, K.; Yoshimura, K.; Kai, T.; Kawada, A.; Miyazaki, H.; Adachi, C. Efficient up-conversion of triplet excitons into a singlet state and its application for organic light emitting diodes. Appl. Phys. Lett. 2011, 98, 083302. [CrossRef]

10. Zhang, Q.S.; Li, B.; Huang, S.P.; Nomura, H.; Tanaka, H.; Adachi, C. Efficient blue organic light-emitting diodes employing thermally activated delayed fluorescence. Nat. Photonics 2014, 8, 326-332. [CrossRef]

11. Cai, X.Y.; Li, X.L.; Xie, G.Z.; He, Z.Z.; Gao, K.; Liu, K.K.; Chen, D.C.; Cao, Y.; Su, S.J. “Rate-limited effect” of reverse intersystem crossing process: The key for tuning thermally activated delayed fluorescence lifetime and efficiency roll-off of organic light emitting diodes. Chem. Sci. 2016, 7, 4264-4275. [CrossRef]

12. Wong, M.Y.; Zysman-Colman, E. Purely organic thermally activated delayed fluorescence materials for organic light-emitting diodes. Adv. Mater. 2017, 29, 54. [CrossRef]

13. Kothavale, S.; Chung, W.J.; Lee, J.Y. High efficiency and long lifetime orange-red thermally activated delayed fluorescent organic light emitting diodes by donor and acceptor engineering. J. Mater. Chem. C 2021, 9, 528-536. [CrossRef]

14. Li, J.; Zhang, Q.S.; Nomura, H.; Miyazaki, H.; Adachi, C. Thermally activated delayed fluorescence from ${ }^{3} \mathrm{n} \pi^{*}$ to ${ }^{1} \mathrm{n} \pi^{*}$ upconversion and its application to organic light-emitting diodes. Appl. Phys. Lett. 2014, 105, 013301. [CrossRef]

15. Li, J.; Gong, H.; Zhang, J.; Zhou, S.; Tao, L.; Jiang, L.; Guo, Q. Enhanced electroluminescence based on a pi-conjugated heptazine derivative by exploiting thermally activated delayed fluorescence. Front. Chem. 2021, 9, 693813. [CrossRef]

16. Goushi, K.; Yoshida, K.; Sato, K.; Adachi, C. Organic light-emitting diodes employing efficient reverse intersystem crossing for triplet-to-singlet state conversion. Nat. Photonics 2012, 6, 253-258. [CrossRef]

17. Li, J.; Nomura, H.; Miyazaki, H.; Adachi, C. Highly efficient exciplex organic light-emitting diodes incorporating a heptazine derivative as an electron acceptor. Chem. Commun. 2014, 50, 6174-6176. [CrossRef] [PubMed]

18. Graves, D.; Jankus, V.; Dias, F.B.; Monkman, A. Photophysical investigation of the thermally activated delayed emission from films of m-mtdata: Pbd exciplex. Adv. Funct. Mater. 2014, 24, 2343-2351. [CrossRef]

19. Liu, X.K.; Chen, Z.; Zheng, C.J.; Liu, C.L.; Lee, C.S.; Li, F.; Ou, X.M.; Zhang, X.H. Prediction and design of efficient exciplex emitters for high-efficiency, thermally activated delayed-fluorescence organic light-emitting diodes. Adv. Mater. 2015, 27, $2378-2383$. [CrossRef]

20. Zhang, M.; Zheng, C.J.; Lin, H.; Tao, S.L. Thermally activated delayed fluorescence exciplex emitters for high-performance organic light-emitting diodes. Mater. Horiz. 2021, 8, 401-425. [CrossRef]

21. Wang, Q.; Ding, J.Q.; Ma, D.G.; Cheng, Y.X.; Wang, L.X.; Jing, X.B.; Wang, F.S. Harvesting excitons via two parallel channels for efficient white organic leds with nearly $100 \%$ internal quantum efficiency: Fabrication and emission-mechanism analysis. Adv. Funct. Mater. 2009, 19, 84-95. [CrossRef] 
22. Li, J.; Nakagawa, T.; MacDonald, J.; Zhang, Q.; Nomura, H.; Miyazaki, H.; Adachi, C. Highly efficient organic light-emitting diode based on a hidden thermally activated delayed fluorescence channel in a heptazine derivative. Adv. Mater. 2013, 25, 3319-3323. [CrossRef]

23. Kumar, S.; Sharma, N.; Kailasam, K. Emergence of s-heptazines: From trichloro-s-heptazine building blocks to functional materials. J. Mater. Chem. A 2018, 6, 21719-21728. [CrossRef]

24. Audebert, P.; Kroke, E.; Posern, C.; Lee, S.H. State of the art in the preparation and properties of molecular monomeric s-heptazines: Syntheses, characteristics, and functional applications. Chem. Rev. 2021, 121, 2515-2544. [CrossRef]

25. Li, J.; Zhang, J.; Gong, H.; Tao, L.; Wang, Y.; Guo, Q. Efficient deep-blue electroluminescence employing heptazine-based thermally activated delayed fluorescence. Photonics 2021, 8, 293. [CrossRef]

26. Li, J.; Tao, L.; Wang, Y.; Yao, Y.; Guo, Q. Heptazine-based $\pi$-conjugated materials for light-emitting. Front. Chem. 2021, 9, 717569. [CrossRef]

27. Hung, W.Y.; Fang, G.C.; Chang, Y.C.; Kuo, T.Y.; Chou, P.T.; Lin, S.W.; Wong, K.T. Highly efficient bilayer interface exciplex for yellow organic light-emitting diode. ACS Appl. Mater. Interfaces 2013, 5, 6826-6831. [CrossRef] [PubMed]

28. Lin, T.; Zhang, T.Y.; Song, Q.G.; Jin, F.M.; Liu, Z.Q.; Su, Z.S.; Luo, Y.S.; Chu, B.; Lee, C.S.; Li, W.L. Thermally activated delayedfluorescence organic light-emitting diodes based on exciplex emitter with high efficiency and low roll-off. Org. Electron. 2016, 38, 69-73. [CrossRef]

29. Yuan, S.L.; Du, X.Y.; Zhao, J.W.; Liu, W.; Lin, H.; Zheng, C.J.; Tao, S.L.; Zhang, X.H. High-performance red organic light-emitting devices based on an exciplex system with thermally activated delayed fluorescence characteristic. Org. Electron. 2016, 39, 10-15. [CrossRef]

30. Santos, P.L.; Dias, F.B.; Monkman, A.P. Investigation of the mechanisms giving rise to tadf in exciplex states. J. Phys. Chem. C 2016, 120, 18259-18267. [CrossRef]

31. Liu, W.; Chen, J.X.; Zheng, C.J.; Wang, K.; Chen, D.Y.; Li, F.; Dong, Y.P.; Lee, C.S.; Ou, X.M.; Zhang, X.H. Novel strategy to develop exciplex emitters for high-performance oleds by employing thermally activated delayed fluorescence materials. Adv. Funct. Mater. 2016, 26, 2002-2008. [CrossRef]

32. Wu, T.L.; Liao, S.Y.; Huang, P.Y.; Hong, Z.S.; Huang, M.P.; Lin, C.C.; Cheng, M.J.; Cheng, C.H. Exciplex organic light-emitting diodes with nearly $20 \%$ external quantum efficiency: Effect of intermolecular steric hindrance between the donor and acceptor pair. ACS Appl. Mater. Interfaces 2019, 11, 19294-19300. [CrossRef] [PubMed]

33. Shirota, Y.; Kageyama, H. Charge carrier transporting molecular materials and their applications in devices. Chem. Rev. 2007, 107, 953-1010. [CrossRef] [PubMed] 